\title{
DrSPINE - New approach to data reduction and analysis for neutron spin echo experiments from pulsed and reactor sources
}

\author{
P.A. Żołnierczuk ${ }^{1,2, a}$, O. Holderer ${ }^{1,3}$, M. Monkenbusch ${ }^{1}$ and M. Ohl ${ }^{1,2}$ \\ 1 Jülich Centre for Neutron Science, Forschungszentrum Jülich GmbH, 52425 Jülich, Germany \\ 2 Jülich Centre for Neutron Science, Outstation at ORNL, Oak Ridge, TN 37831, USA \\ 3 Jülich Centre for Neutron Science, Outstation at MLZ, 85747 Garching, Germany
}

\begin{abstract}
Neutron spin echo (NSE) method at a pulsed neutron source presents new challenges to the data reduction and analysis as compared to the instruments installed at reactor sources. The main advantage of the pulsed source NSE is the ability to resolve the neutron wavelength and collect neutrons over a wider bandwidth. This allows us to more precisely determine the symmetry phase and measure the data for several $Q$-values at the same time. Based on the experience gained at the SNS NSE - the first, and to date the only one, NSE instrument installed at a pulsed spallation source, we propose a novel and unified approach to the NSE data processing.
\end{abstract}

\section{Introduction}

Neutron spin echo spectroscopy has become a standard technique for condensed matter research $[1,2]$ and with the advent of spallation sources it has been successfully adopted to the pulsed source environment [3].

Jülich Centre for Neutron Science (JCNS) operates two NSE instruments: one at the Meier-Leibnitz Zentrum (MLZ) in Garching, Germany [4] and the other one at the Spallation Neutron Source (SNS) in Oak Ridge, TN, USA [3], see Fig. 1. The chief difference between an NSE instrument installed at a pulsed source versus a one installed at a reactor is the ability to resolve the neutron wavelength via the time-of-flight method (TOF). Also the simultaneously available bandwidth is typically larger at a pulsed source ${ }^{1}$.

Different versions of the original reduction software echodet [5] are currently used at the two JCNS instruments making it harder to incorporate new algorithms, new reduction strategies and debugging the software. It is also more difficult to compare the data taken at different instruments. Moreover, the pulsed source evaluation version is essentially a patch on the original reactor code that does not fully take into account the available time-offlight information.

\section{DrSPINE design}

In order to address the above mentioned issues a collaboration of JCNS instrument scientists was formed with the aim to produce a uniform software solution capable of reducing the data from both instruments and potentially from other instruments, either existing (e.g. NIST NG5) or planned (e.g. at the European Spallation Source).

\footnotetext{
a e-mail: p.zolnierczuk@fz-juelich.de

${ }^{1}$ At reactor instruments larger bandwidth may be covered by sequential experiments.
}

The main goals of the DrSPINE ${ }^{2}$ project are:

- exploit better symmetry phase determination due to the broader bandwidth at a pulsed source, see Fig. 2.

- take advantage of larger $Q$ coverage for TOF instruments, see Fig. 3 and Fig. 4.

- use objective statistical criteria to get the echo fits right

- provide robust reduction with report generation

- incorporate absolute instrument calibration

- allow for background subtraction.

The software must be able to read the data from various instruments, perform data integrity, consistency and compatibility checks and combine the data from compatible sets, partial scans, etc. We chose to provide a console-based interface with the ability to process macros (scripts) for batch evaluation. And last and not the least, a good software package has to provide adequate documentation.

\section{Use cases}

Here we outline typical use cases that we propose to address.

\subsection{Automatic reduction}

This is the default use case providing fully automatic (unattended) evaluation of an NSE experiment with comprehensible error and diagnostic messages. The evaluation process is outlined as follows:

1. Specification of the data files

(a) read one or more reference files

(b) read one or more data files

(c) read background files (if provided)

${ }^{2}$ DrSPINE - Data Reduction for SPIN Echo experiments.

This is an Open Access article distributed under the terms of the Creative Commons Attribution License 4.0, which permits unrestricted use, distribution, and reproduction in any medium, provided the original work is properly cited. 


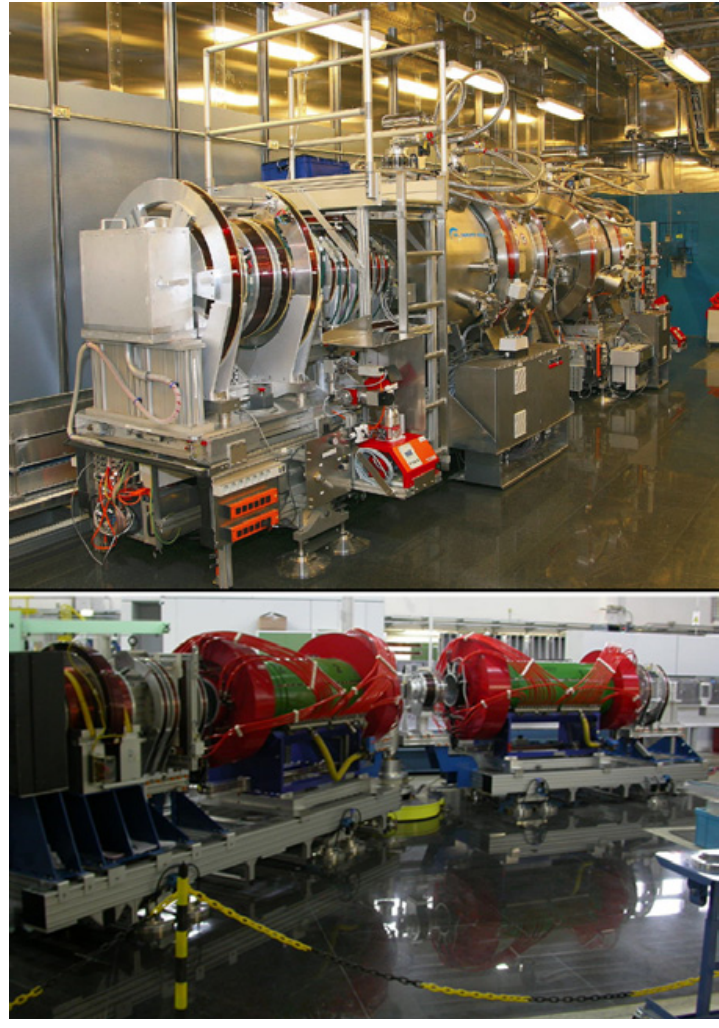

Figure 1. Neutron spin echo instruments at pulsed and reactor sources. The top photo shows the SNS-NSE instrument at the Spallation Neutron Source (SNS), whereas the bottom one shows the J-NSE instrument at the Meier-Leibnitz Zentrum (MLZ).

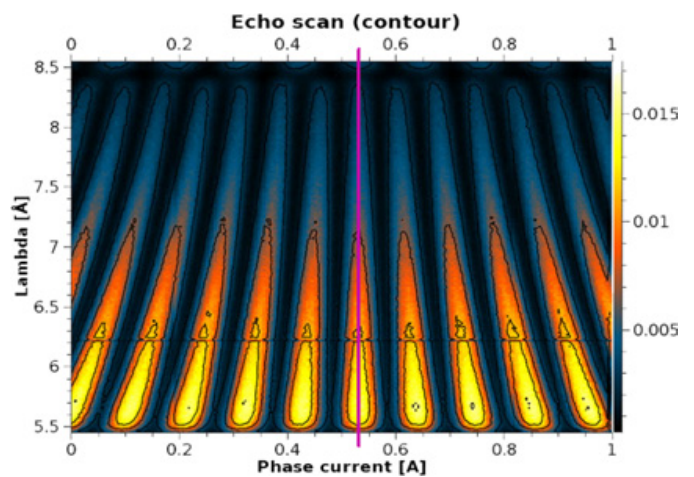

Figure 2. Wavelength bandwidth covered at an NSE experiment carried out at a pulsed source. Note that one typically measures only around the symmetry phase (magenta vertical line) that does not depend on the incoming neutron wavelength.

(d) read calibration file (if available)

(e) read $S(Q)($ resp. $I(Q))$, diffraction run or from other instruments (optional step).

2. Specification of the evaluation process

(a) parameters/options controlling the evaluation processes

(b) detector masks/mask strategy

(c) data binning.

3. Run the evaluation

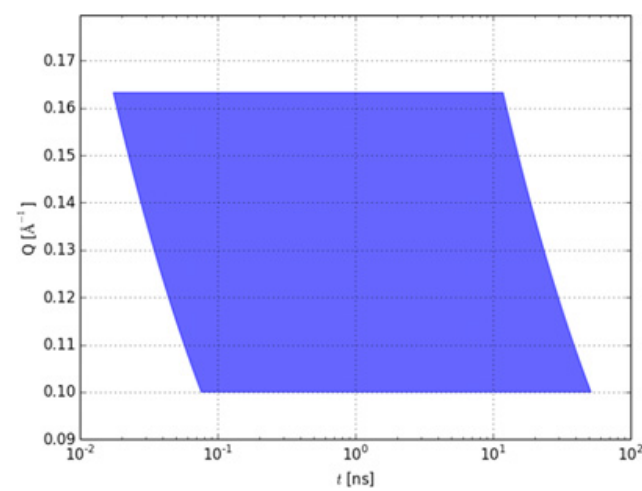

Figure 3. The $Q-t$ coverage for a center of the SNS-NSE detector at $\lambda=5-8 \AA$ and $2 \Theta=7.3^{\circ}$, corresponding to $Q_{\min }=$ $0.1 \AA^{-1}$.

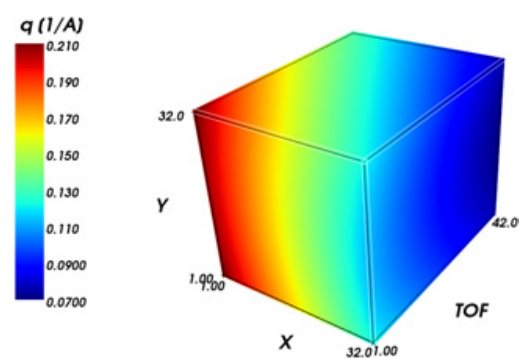

Figure 4. The $Q$ coverage for the entire SNS-NSE detector at $\lambda=5-8 \AA$ and $2 \Theta=7.3^{\circ}$, corresponding to $Q_{\min }=0.1 \AA^{-1}$ at the center of the detector. The binning is done for a typical SNS-NSE set-up of $32 \times 32$ pixels and 42 TOF channels.

\section{Final steps}

(a) check the reduction protocol

(b) collect the data.

\subsection{Symmetry phase refinement}

Primary source for symmetry phases, e.g. in terms of field integral difference $\Delta J$ or phase coil currents, are fits to the reference data which has sufficiently good statistics. The symmetry phase depends on: a) field integral $J=$ $\int B d l$, i.e. current settings, $\mathrm{b}$ ) scattering angle, c) detector pixel position and d) environmental magnetic fields. The symmetry phase does not depend on the wavelength (see Fig. 2) as long as the currents are held constant. The above fact is utilized to improve the phase accuracy by including the full wavelength band into the phase determination (see Fig. 5).

For the symmetry phases of the sample or background scans, a selection must be made between:

- taking the reference phases

- taking the reference phases with one common offset determined by fitting the sample (background) data

- individual fits with references phases as starting values.

The first method is prone to errors induced by external magnetic fields, whereas the phase fits to sample and especially background data may become very difficult or even impossible if the echo signal is small or noisy. The objective statistical criteria will guide the selection of the optimal method. 


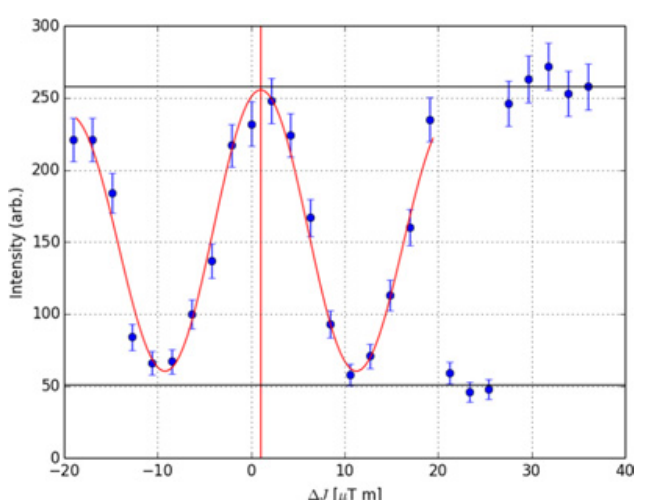

Figure 5. Precise symmetry phase determination (red vertical line) is the crucial and the central point of each NSE evaluation procedure.

\subsection{Prior knowledge of phases}

Instrument scientist supplies data with phase-maps, e.g. determined with some high quality reference data, possibly edits the maps, and then uses them as input for the echo phase fit determination. This will be used typically in fixing fitting problems, i.e. when automatic evaluation fails.

\subsection{SANS data aided reduction}

User provides SANS measurements data that can, for example, be used to:

- compute the scattering function as follows: $S(Q, t)=$ $S(Q)_{\text {SANS }} \times\left(\frac{S(Q, t)}{S(Q)}\right)_{\mathrm{NSE}}$.

- infer intensity calibration

- compute the echo shape function.

\subsection{Instrument scientist reduction}

This use case involves detailed ("debug") evaluation that allows for interactive and atomic execution of each evaluation step.

\section{Current status and outlook}

DrSPINE is currently under development. The input (data reading) module as well as the "fitting engine" are under testing (see Fig. 6).

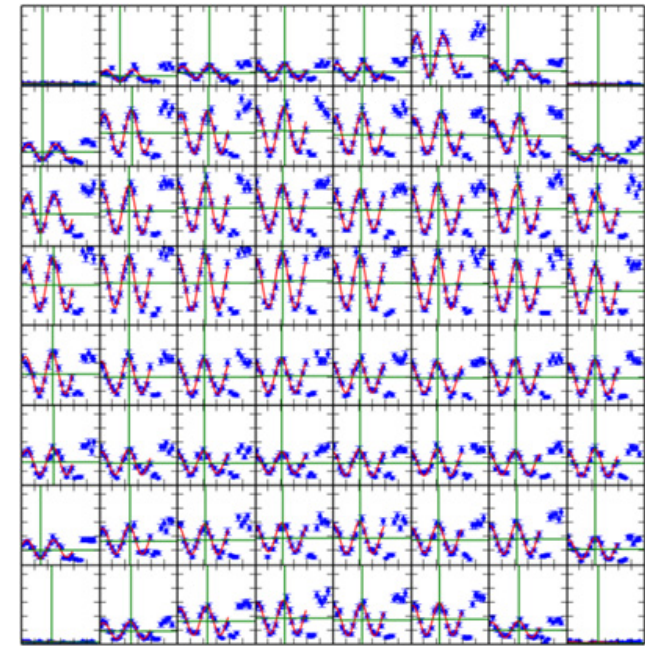

Figure 6. Preliminary echo fits to the SNS-NSE resolution data (grafoil sample) obtained with an early version of DrSPINE. The figure shows pixel fits for data summed over all available neutron wavelengths.

We strive to provide a software package that will allow for automatic conversion of the NSE raw data to $S(Q, t)$ with the ability to (simultaneously) take advantage of all information contained in the whole collection of scans pertaining the scientific problem under consideration and possibly combining data from different instruments. DrSPINE will generate comprehensive reports, concise summary and easy to use formats for $S(Q, t)$. It will permit (as far as it is possible) the decomposition of coherent and incoherent contribution, enable automatic choice of optimal evaluation methods, proper error determination, identification of problems that may have occurred during the measurement (e.g. variation of intensity) and the expert mode to further analyze problematic data.

\section{References}

[1] F. Mezei, Z. Physik 255, 146 (1972)

[2] F. Mezei, C. Pappas, T. Gutverlet (Eds.), Neutron Spin Echo Spectroscopy. Basics, Trends and Applications (Springer-Verlag, 2003)

[3] M. Ohl et al., Nucl. Inst. and Meth. A 696, 85-99 (2012)

[4] O. Holderer et al., Nucl. Inst. and Meth. A 586, 90-94 (2008)

[5] M. Monkenbusch, priv. comm. 